\title{
Mixoma auricular izquierdo gigante que causa obstrucción valvular mitral, hipertensión pulmonar y tromboembolia pulmonar
}

\author{
Cristián Olmedo ${ }^{1}$, Juan Álvarez ${ }^{2}$, Fernando Rodríguez ${ }^{3}$, Carlos Ramírez $^{3}$, Hernaldo Veloz $^{3}$, Constanza Castro ${ }^{2}$, Eric Fuentes ${ }^{2}$, Juan Lahsen ${ }^{4}$. \\ 1. Residente de Cardiología, Universidad Católica del Maule. \\ 2. Cardiólogo, CR Cardiología y cirugía cardiovascular del Hospital Regional de Talca. \\ 3. Cirujano Cardíaco, CR Cardiología y cirugía cardiovascular del Hospital Regional de Talca. \\ 4. Anatomo - patólogo, Unidad de Anatomía patológica del Hospital Regional de Talca. \\ Institución: Hospital Regional de Talca. \\ Financiamiento: Ninguno.
}

Los tumores cardíacos pueden ser primarios o, más frecuentemente secundarios o metastásicos. Entre los tumores primarios es más frecuente el mixoma, cuya ubicación más común es en la aurícula izquierda. Las manifestaciones clínicas son diversas, producidas principalmente por obstrucción mecánica, embolizaciones, y manifestaciones constitucionales.

Se comunica el caso de un paciente de 32 años, con cuadro clínico de insuficiencia cardíaca, hipertensión pulmonar severa y tromboembolismo pulmonar bi- lateral. Se hizo el diagnóstico de mixoma auricular izquierdo. Se resecó el tumor y se manejó la hipertensión pulmonar desde el ingreso al hospital con inhibidores de la fosfodiesterasa asociado a anticoagulación. Se discute el tema dando énfasis a aspectos fisiopatológicos involucrados tanto en la hipertensión pulmonar como en la presencia de tromboembolia pulmonar.

Palabras clave: mixoma auricular; hipertensión pulmonar; tromboembolismo pulmonar.

\section{Giant atrial myxoma causing mitral valve obstruction, pulmonary hypertension and pulmonary thromboembolism}

Cardiac tumors may be primary or, more frequently secondary or associated to metastasis. Atril myxoma es the most frequent primary tumor, usually located in the left atrium. Clinical manifestations include those due to mitral valve occlusión, emboli and general non spedific symptoms and signs. Herein we report the clinical case of a 32 year old patient with severe plmonary hyper- tension and bilateral pulmonary embolism. The tumor was extirpated, and he received phosphoro-diesterase inhiborts and anticoagulants. Subsequent clinical course was satisfactory. A brief discussion of this condicion is included.

Keywords: mixoma, atrial; pulmonary hypertension; thromboembolism, pulmonary. 
El mixoma es un tumor cardíaco benigno que se aloja principalmente en la aurícula izquierda. Tiene diversas manifestaciones clínicas que incluyen obstrucción valvular e hipertensión pulmonar, las cuales dependen de la ubicación y el tamaño del tumor. El tratamiento es principalmente quirúrgico. Reportamos un caso de mixoma auricular izquierdo con manifestaciones como las descritas en un paciente de 32 años.

\section{Caso Clínico:}

Paciente de sexo masculino de 32 años de edad, de nacionalidad haitiana, sin antecedentes mórbidos conocidos que consulta en el departamento de emergencias por cuadro de disnea de esfuerzo de un mes de evolución, asociada a tos seca y edema de extremidades inferiores. Al examen físico se constata taquicárdico con frecuencia cardíaca de $100 \mathrm{lpm}$, normotenso (115/82 mmHg), con ingurgitación yugular, sin hallazgos de relevancia a la auscultación cardíaca, con crepitaciones finas en bases pulmonares, abdomen globuloso con sensibilidad a la palpación en hipocondrio derecho y edema de extremidades inferiores. El electrocardiograma muestra

Figura 1: Imágenes por ecocardiograma transtorácico.

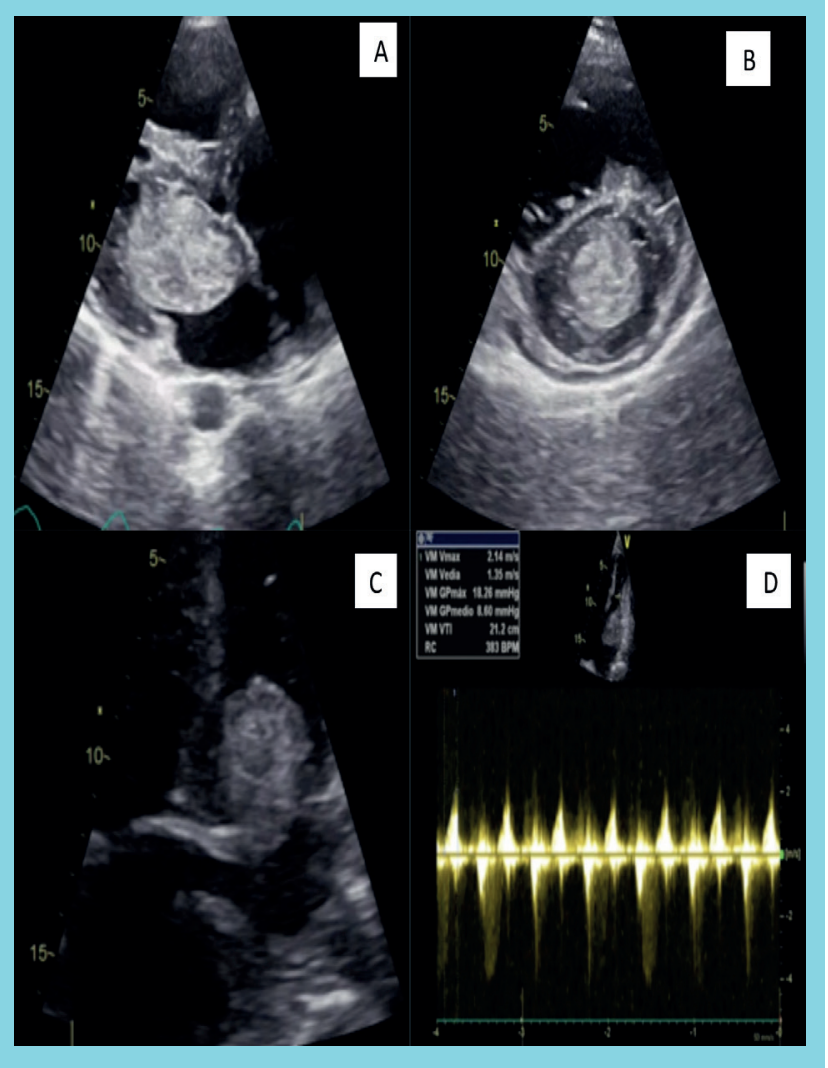

taquicardia sinusal con crecimiento auricular izquierdo y signos de crecimiento y sobrecarga ventricular derecha. En la radiografía de tórax se evidencia cardiomegalia y signos de congestión pulmonar. Los exámenes de laboratorio mostraron creatinina $1.16 \mathrm{mg} / \mathrm{dl}$, bilirrubina total $3.67 \mathrm{mg} / \mathrm{dl}$, bilirrubina directa $2.92 \mathrm{mg} / \mathrm{dl}$, Fosfatasas alcalinas 118 UI/L, GOT 329 UI/L, GPT 179 UI/L, natremia $133 \mathrm{meq} / 1$, PCR $62.1 \mathrm{mg} / \mathrm{L}, \mathrm{LDH} 629 \mathrm{UI} / \mathrm{L}$, Pro BNP 3006 pg/ml, Albumina 2.9 g/dl, hematocrito $37.2 \%$, hemoglobina $11.5 \mathrm{~g} / \mathrm{dl}$, VCM 76 fl, CHCM 30.9 g/dl, HCM 23.5, leucocitos 8790 por mm3, Plaquetas 201000 por mm3, VHS 2. Además, presencia elevada de carboxihemoglobina (2.5\%) El ecocardiograma transtorácico reveló ventrículo izquierdo no dilatado con función sistólica normal (FE 63\%), dilatación severa biauricular, con volumen indexado de aurícula izquierda de 48 $\mathrm{ml} / \mathrm{m} 2$. En su interior se observó una imagen de masa pediculada de 5.8 × $3.4 \mathrm{~cm}$, insertada en el septo interauricular, cerca del anillo valvular mitral, que prolapsa a través se válvula mitral durante la diástole, llegando hasta nivel medio ventricular, asociada a una gradiente transvalvular mitral moderada (Figura 1-A, 1-B, 1-C, 1-D). Además, se evidencia dilatación del ventrículo derecho con función sistólica limítrofe (TAPSE $17 \mathrm{~mm}$ y onda $\mathrm{S}$ lateral del anillo tricuspídeo de $12 \mathrm{~cm} / \mathrm{s}$ ), insuficiencia tricuspídea severa e hipertensión pulmonar severa con PSAP de $89 \mathrm{mmHg}$ (Figura 2). El TAC de tórax evidencia cardiomegalia, dilatación de arteria pulmonar, derrame pleural bilateral (Figura 3-A), imágenes en vidrio esmerilado en ambos pulmones (Figura 3-B) e imagen tumoral intracardíaca (Figura 3-C). El angio TAC de tórax revela tromboembolismo pulmonar subsegmentario subagudo bilateral (Figura 3-D) y trombo en vena yugular interna derecha. En el TAC de abdomen se evidencia aumento del tamaño renal e imágenes quísticas hepáticas asociado a hepatomegalia (Figura 3-E). Se maneja inicialmente con diuréticos de ASA e inhibidores de la fosfodiesterasa 5.Al sexto día del ingreso se realiza resección de la masa y su pedículo, cerrando el defecto en el tabique interauricular con parche de pericardio bovino. La tumoración resecada es de aspecto gelatinoso de 6 x $5 \mathrm{~cm}$ (Figura 4-A) evidenciándose en el análisis histológico presencia de tejido mixomatoso Figura 4-B). Durante el post operatorio, se mantiene con apoyo de ventilación mecánica invasiva que se prolonga por cinco días, tratamiento inotrópico con milrinona que se suspende al octavo día, vasopresores durante las primeras 24 hrs., e inhibidores de la fosfodiesterasa 5 que se mantienen hasta el alta. Durante el post operatorio inmediato, 
Figura 2: llustra la gradiente de presión transmitral.

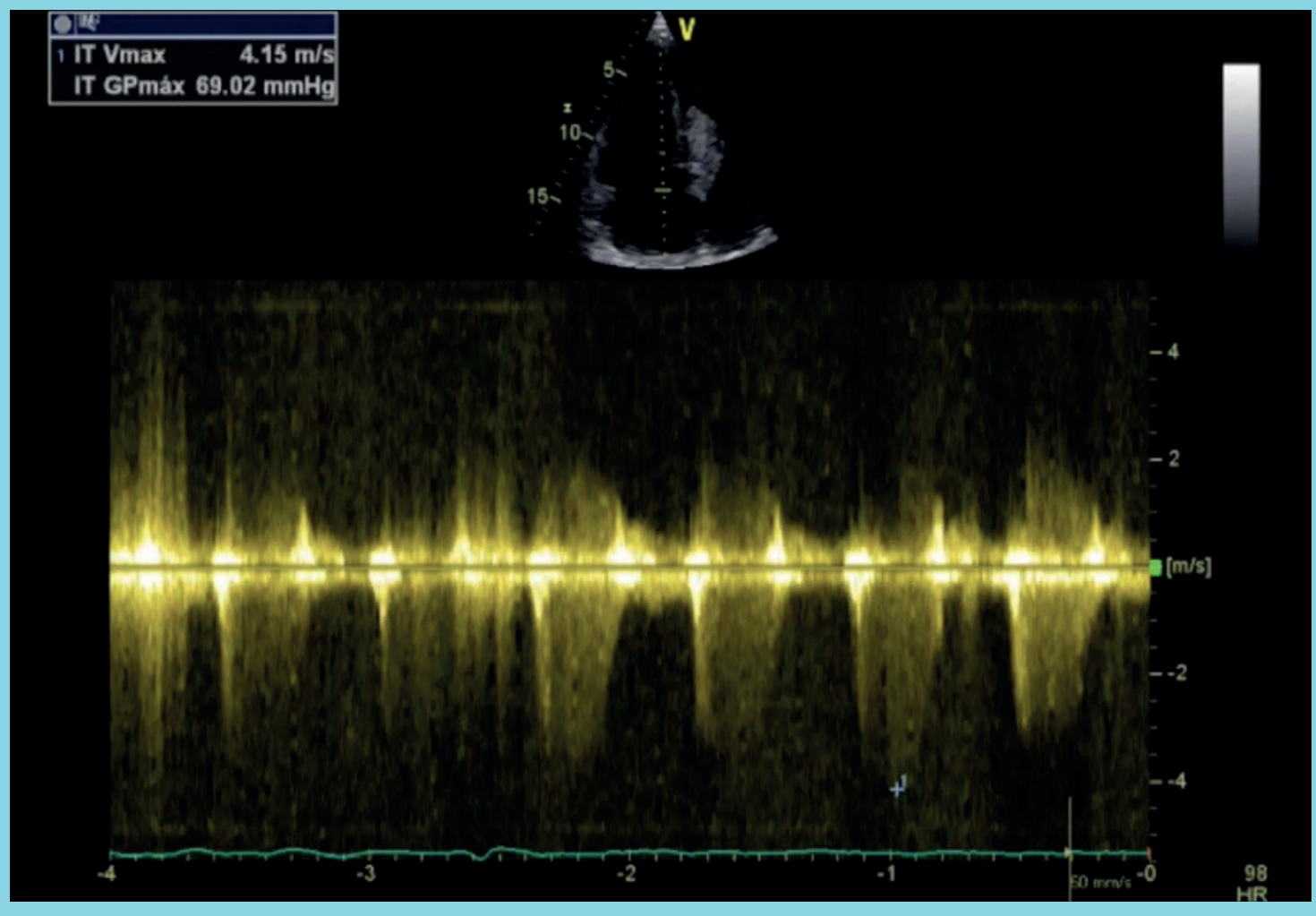

Figura 3: Tomografía axial computarizada de tórax, angio TAC de tórax y TAC de tórax yb BDOMEN abdomen (explicaicón en el texto).

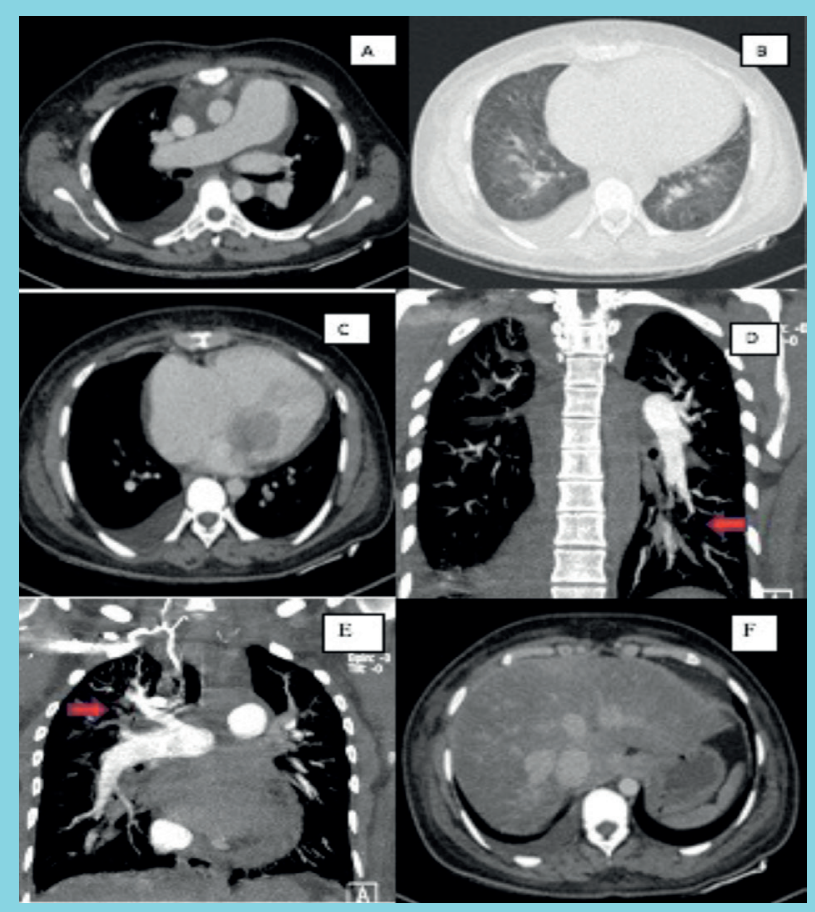

la presión en arteria pulmonar medida por catéter Swan Ganz mostró PSAP 74 mmHg, PDAP 31 mmHg, PMAP $43 \mathrm{mmHg}$. A los 11 días post cirugía la PSAP evaluada por ecocardiograma transtorácico fue $51 \mathrm{mmHg}$. Además, en la evaluación ecocardiográfica post quirúrgica se destacó el regreso a la normalidad de las dimensiones de la aurícula izquierda.

Al día 14 de la cirugía el paciente es dado de alta, con inhibidores de la fosfodiesterasa 5, diuréticos de ASA, antagonistas de receptores de aldosterona, IECA en dosis bajas y anticoagulación oral con antagonistas de la vitamina $\mathrm{K}$.

\section{Discusión:}

Los tumores cardíacos son raros, sin embargo su conocimiento es importante dadas sus implicaciones terapéuticas. Abarcan un amplio espectro de lesiones que pueden ser clasificadas en neoplásicas y no neoplásicas. Las neoplásicas pueden ser clasificadas a su vez en primarias o secundarias (metástasis al corazón), siendo estas últimas las más frecuentes. La incidencia de tumores cardíacos primarios diagnosticados clínicamente es de 1.380/100 millones de personas. Comparados con tumores cardíacos primarios, los secundarios tienen una incidencia de 

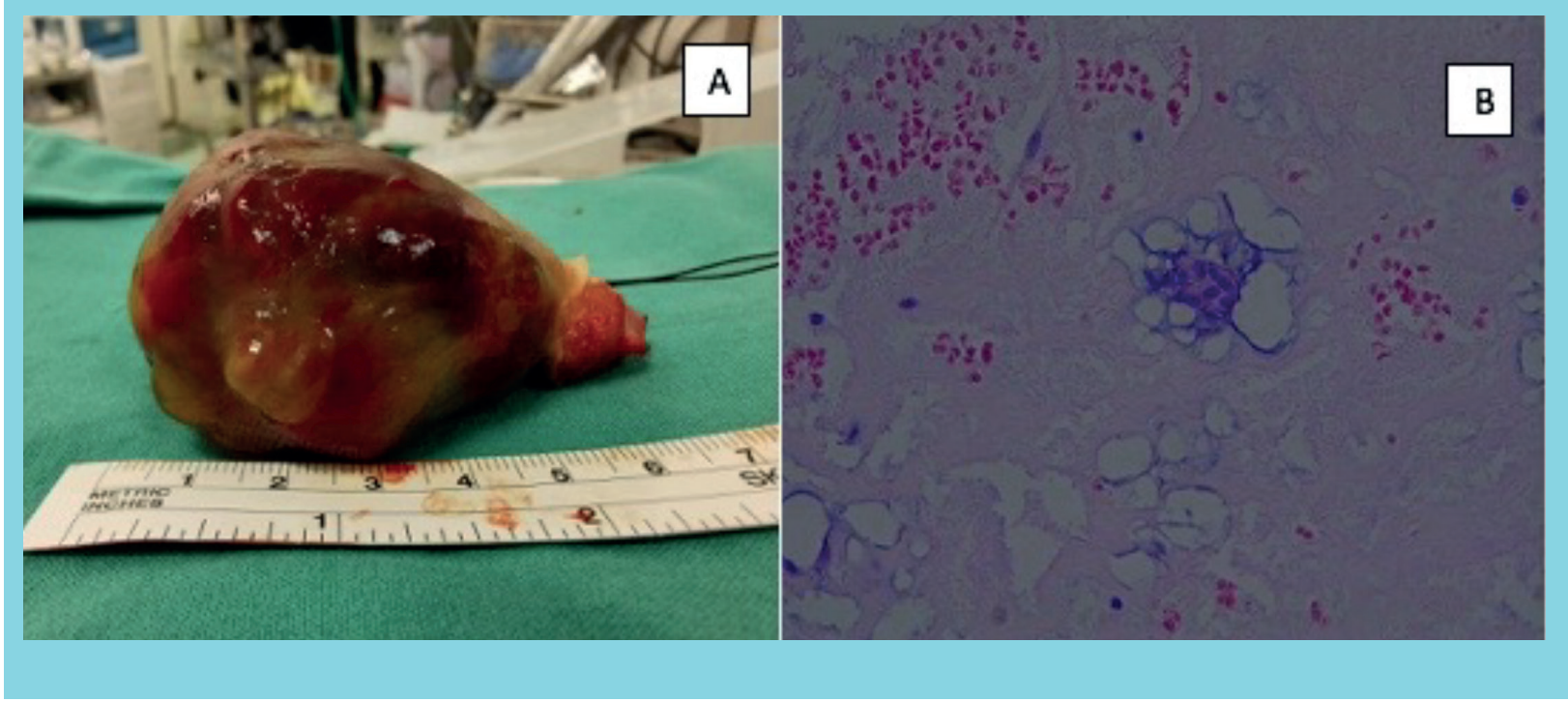

22 a 132 veces mayor, siendo habitualmente malignos. El $90 \%$ de los tumores cardíacos primarios son benignos, originados principalmente en el miocardio o pericardio. La clasificación de las lesiones como benignas o malignas tiene importantes implicancias pronósticas. Sin embargo, a pesar de que un tumor cardíaco sea histológicamente benigno, dependiente de su tamaño y localización, puede tener repercusiones hemodinámicas y arrítmicas ${ }^{1}$.

Los mixomas pueden presentarse en cualquier etapa de la vida, siendo más frecuente entre la tercera y sexta déca$\mathrm{da}^{2}$. Habitualmente, se presentan de forma esporádica, sin embargo, existen casos en los que se presenta con patrón familiar $^{3}$. La ubicación más frecuente es la aurícula izquierda ( $75 \%$ de los casos), seguida por la aurícula derecha $(15-20 \%)$, ventrículo derecho (3 a 4\%) y ventrículo izquierdo (3-4\%). Pocos casos son múltiples ya sea en la misma aurícula o en distintos sitios, siendo esto más frecuente en los casos familiares. Usualmente se desarrollan desde el borde de la fosa oval, ubicada en el septo interauricular, pudiendo originarse con menor frecuencia en pared anterior o posterior de la auricula ${ }^{4}$.

La presentación clínica se relaciona con la ubicación y el tamaño del mixoma. Puede variar desde asintomático en caso de tumores de pequeño tamaño hasta la muerte súbita. Los síntomas clásicos se relacionan con embolia, síntomas y signos derivados de obstrucción intracardíaca con alteraciones hemodinámicas y síntomas constitucionales. La embolia se puede observar hasta en 30 a $40 \%$ de los casos, siendo principalmente a sistema nervioso central. Los signos derivados de obstrucción intracardíaca son principalmente derivados de la obstrucción del llenado de las cavidades ventriculares, presentándose con disnea, edema pulmonar recurrente o insuficiencia cardíaca derecha. Los signos constitucionales o generales, son diversos entre los que se pueden encontrar artralgias, fiebre, astenia, rash eritematoso, o alteraciones de laboratorio como anemia habitualmente normocítica normocrómica, siendo en algunos casos hemolítica por destrucción de eritrocitos por la masa, elevación de la velocidad de eritrosedimentación, policitemia, trombocitosis o cianosis. Puede simular enfermedades infecciosas, inflamatorias o neoplásicas, atribuyéndose esto a producción por el tumor de mediadores pro inflamatorios como interleucina 65. La presencia de fenómenos tromboembólicos paradojales se ha descrito en reportes de casos de mixomas atriales izquierdos asociados con la presencia de foramen oval permeable como principal causa ${ }^{6}$, y en ausencia de esto, secundario a una actividad aumentada de la enzima Heme - oxigenasa - 1, la que produce un aumento de la carboxihemoglobina utilizando el monóxido de carbono endógeno y heme libre, sustrato que puede encontrarse aumentado por el flujo turbulento que produce el mixoma con la consecuente hemolisis en grado variable. La carboxihemoglobina formada al reaccionar con fibrinógeno forma carboxihemefibrinógeno, responsable de la hipercoagulabilidad e hipofibrinolisis ${ }^{7}$.

El tratamiento de elección es la extirpación quirúrgica del tumor, evitando así complicaciones embólicas o muerte 
súbita. La mortalidad operatoria varía entre un 0 a un $3 \%$ según distintas series, requiriendo muchas veces la utilización de un parche de pericardio o dacrón para solucionar el defecto septal residual ${ }^{8}$. Ocasionalmente, el daño valvular hace necesaria la reparación mitral. La recurrencia es poco frecuente siendo del $3 \%$ en el caso de mixomas esporádicos y del 12 a $22 \%$ en casos familiares 9 .

Se describe en algunos casos la regresión de la dilatación de la aurícula izquierda por remodelado inverso poste- rior a la cirugía de valvulopatía mitral, no encontrándose datos en el contexto de obstrucción al flujo de entrada del ventrículo izquierdo por tumores. El mecanismo fisiopatológico de la dilatación de la aurícula izquierda es la obstrucción mecánica y por la frecuente presencia de fibrilación auricular, con remodelado eléctrico subsecuente. Posterior a la cirugía, se produce frecuentemente remodelado inverso post quirúrgico si no hay fibrilación auricular permanente ${ }^{10}$.

\section{Referencias}

1. SARA TYEBALLY, DANIEL CHEN, SANJEEV BHATTACHARYYA, ABDALLAH MUGHRABI, ZEESHAN HUSSAIN, CHARLOTTE MANISTY, MARK WESTWOOD, ARJUN K. Ghosh, Avirup Guha, J Am Coll Cardiol CardioOnc. 2020 Jun, 2 (2) 293-311.

2. HEATH D. Pathology of cardiac tumors. Am J Cardiol 1968;21:315-27.

3. LIEBLER GA, MAGOVERN GJ, PARK SB, CUSHING WJ, BEGG FR, JOYNER CR. Familial myxomas in four siblings. J Thorac Cardiovasc Surg 1976;71:605-8.

4. MCALLISTER HA JR, FENOGLIO JJ JR. Tumors of the cardiovascular system. Atlas of tumor pathology. 2nd series. Fascicle 15. Washington, D.C.: Armed Forces Institute of Pathology, 1978:1-20.

5. REYNENK.Cardiacmyxomas.NEnglJMed.1995;333(24):16101617. doi:10.1056/NEJM199512143332407.

6. NAGELE R, BONETTI PO, MAYER KA. A patient with pulmonary embolism and a mass in the left atrium. Heart
2012; 98:1260.

7. REDFORD DT, THOMPSON JL, MCCULLOCH JC, NIELSEN VG. Left atrial myxoma presenting as pulmonary embolism: potential role of heme oxygenase-1. Blood Coagul Fibrinolysis. 2014;25(6):621-624. doi:10.1097/ MBC.0000000000000097

8. LIVI U, BORTOLOTTI U, MILANO A, et al. Cardiac myxomas: results of 14 years' experience. Thorac Cardiovasc Surg 1984;32:143-7.

9. WALLER DA, ETTLES DF, SAUNDERS NR, WILLIAMS G. Recurrent cardiac myxoma: the surgical implications of two distinct groups of patients. Thorac Cardiovasc Surg 1989; 37:226-30.

10. HORNERO SOS F, MONTERO ARGUDO JA, CANOVAS LÓPEZ S, et al. Remodelado anatómico auricular tras la cirugía de la valvulopatía mitral con fibrilación auricular permanente. Rev Esp Cardiol. 2003; 56(7): 674-681. doi:10.1016/ s0300-8932(03)76938-3. 